\title{
TMJ Disorders Vs Orthodontic Treatment A Research Study
}

\author{
BL. Abiraamasri*,Dr. Saravanapandian**
}

*IIIrd Yr BDS, Saveetha Dental College And Hospitals

**Senior Lecturer, Saveetha Dental College And Hospitals

\begin{abstract}
The aim of the study is to evaluate the prevalence of TMJ disorders among the group of individuals who are undergoing orthodontic treatment. The sample comprised 100 individuals (59 males and 41 females) are divided into groups according to malocclusion (class I and II). An anamnestic questionnaire, comprising questions regarding the most frequent symptoms of TMJ disorders, was used to classify the samples according to the TMD

(TMJ disorders) presence and severity. A clinical examination, including TMJ and muscle palpation and joint noise analysis was performed.
\end{abstract}

Keywords:TMJ disorders, orthodontic treatment, TMD prevalence malocclusion

\section{Introduction}

Temporo-mandibular disorders (TMD) are musculoskeletal disorders affecting the temporomandibular joint (TMJ), the masticatory muscles or both and they are the most common cause of orofacial somatic nonodontogenic pain(1). The prevalence of the signs and symptoms of temporo-mandibular disorders (TMD) has increased considerably in the past decades. The higher frequency of unavoidable factors like emotional stress plus the availability of accurate diagnostic tests can account for this fact. Many theories regarding TMD have been proposed but the real etiologic factor has not been detected(2). Different therapies like orthodontic or orthopedic treatments as well as occlusal adjustment have been proposed to correct malocclusion and improve TMD signs and symptoms.According to various theories functional and morphological malocclusion cause TMD, and achievement of an ideal occlusion through orthodontic treatment must eliminate signs and symptoms. Orthodontic treatment as a possible TMD etiological factor and considered as the main cause for pain(3).

According to Wyatt, certain orthodontic procedure may increase the risk of developing TMD. The procedures include use of class II elastic and headgear as well as elastics to correct midline deviation, chin cups and retention appliances, these procedures would displace the disk-condyle complex posteriorly and initiates pathological problem in the joint(4). According to Nielsen et al, muscular and TMJ tenderness to palpation was higher in the treated group than in untreated group, but both the groups showed a similar frequency of joint sounds(5)

\section{Materials And Method}

A sample of 100 individuals (59 males and 41 females) was selected from patients of the Department of orthodontics of Saveetha Dental College and hospital. Exclusive criteria were the presences of systemic arthritis and previous treatment of TMD symptoms(6). The individuals were divided into two groups, according to the malocclusion and the orthodontic treatment accomplished. Group I were patients with untreated class I and II malocclusion and group II were patients with treated class I and II malocclusion through orthodontic treatment(7).For the whole sample mouth opening, overjet and overbite were measured and the whole sample was asked to answer an Anamnestic Questionnaire to classify them according to the signs and symptoms of TMD(8).

Questions are related to emotional stress, awareness of dysfunction and habits included in the question were bruxing, clenching and nail biting(9).A score was given for the every response of the patients to questions, a grade of 0 given to absence of symptoms, grade 1 to mild presence of symptoms and grade 3 was used to indicate severe pain or moderate symptoms(10).

The sum of the scores was used to classify the sample into four categories are

(1) From 0-3 TMD free

(2) From 4-8 mild TMD

(3) From 9-14 moderate TMD

(4) From 15-23 severe TMD

\section{Anamnestic Questionnaire}

1) Do you have difficulty in opening your mouth?

2) Do you have difficulty in moving your jaw?

3) Do you have muscular tenderness?

4) Do you have frequent headaches?

5) Do you have shoulder pain?

6) Do you have pain in or around the ear?

7) Are you aware of noises in the jaw joints?

8) Do you consider your bite normal?

9) Do you use only one side of your mouth when chewing?

10) Do you have morning facial pain?

\section{Result}

When the TMD anamnestic index for the whole sample was considered, $63 \%$ were considered TMD free, $32 \%$ of the subjects had mild TMD and 5\% had moderate TMD (Fig.1). There is no significant difference between group I and group II and no patients was classified to have severe TMD.For the whole sample the mean mouth opening was $36.17 \mathrm{~mm}$, mean overjet was $6.72 \mathrm{~mm}$ and mean overbite was $5.82 \mathrm{~mm}$. 


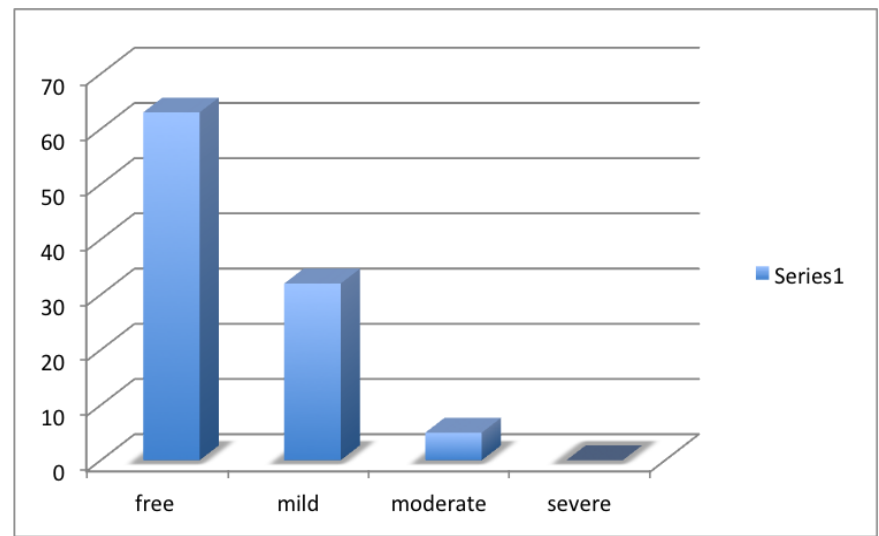

(Fig.1)Chart based on signs and symptoms of the patients

When comparing group I and II, in group I 38\% of patients were TMD free and group II 25\% of patients were TMD free. 10\% of patients had mild TMD in group I and $22 \%$ of patients in group II. $2 \%$ of patients had moderate TMD in group I and $3 \%$ of patients in group II.

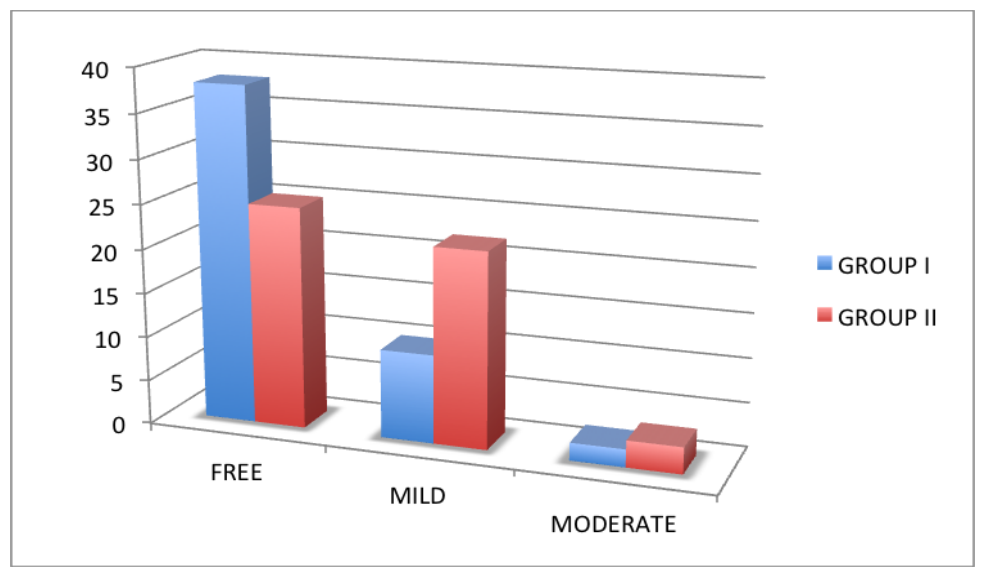

(Fig.2) Chart comparing the signs and symptoms between group I and group II

When comparing the TMD prevalence rate between males and females, there is a very small difference. $64 \%$ of males were considered TMD free and $63 \%$ of females were considered TMD free (Fig.3)

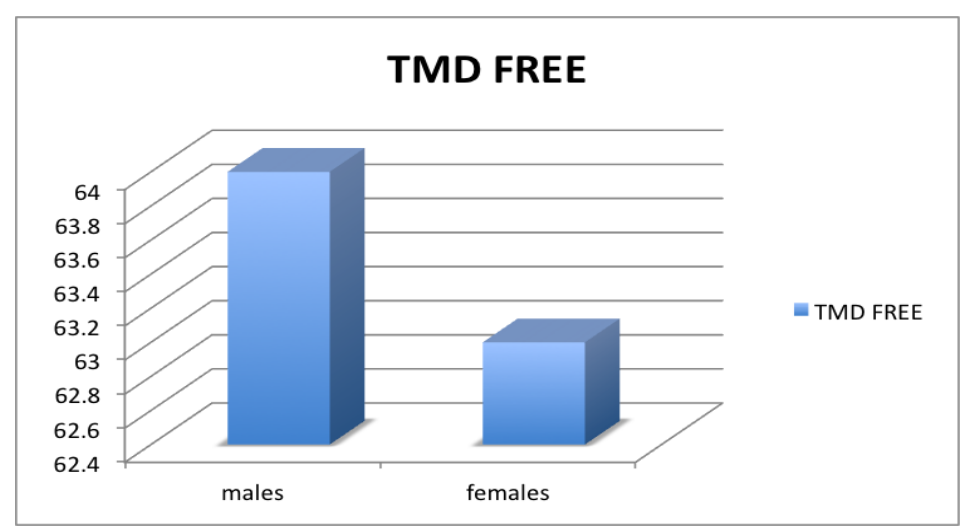

(Fig.3) Comparing TMD free among males and females

Comparing mild TMD between males and females, 31\% of males had mild TMD and 32\% of females had mild TMD(Fig.4) 


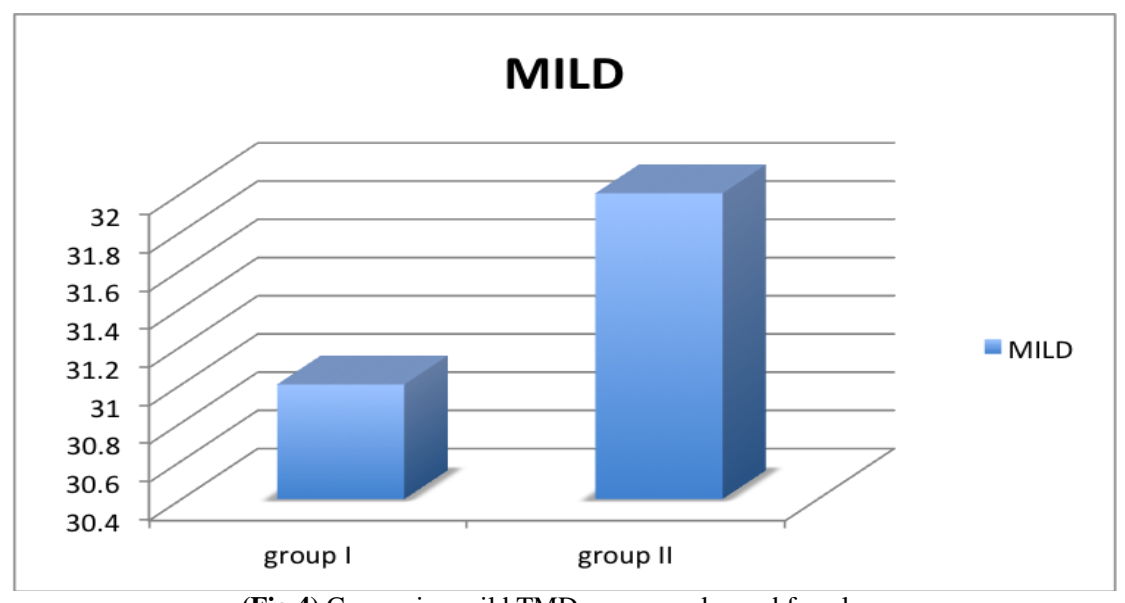

(Fig.4) Comparing mild TMD among males and females.

Among males and females, 3\% of males had moderate TMD and 4\% of females had moderate TMD(Fig.5)

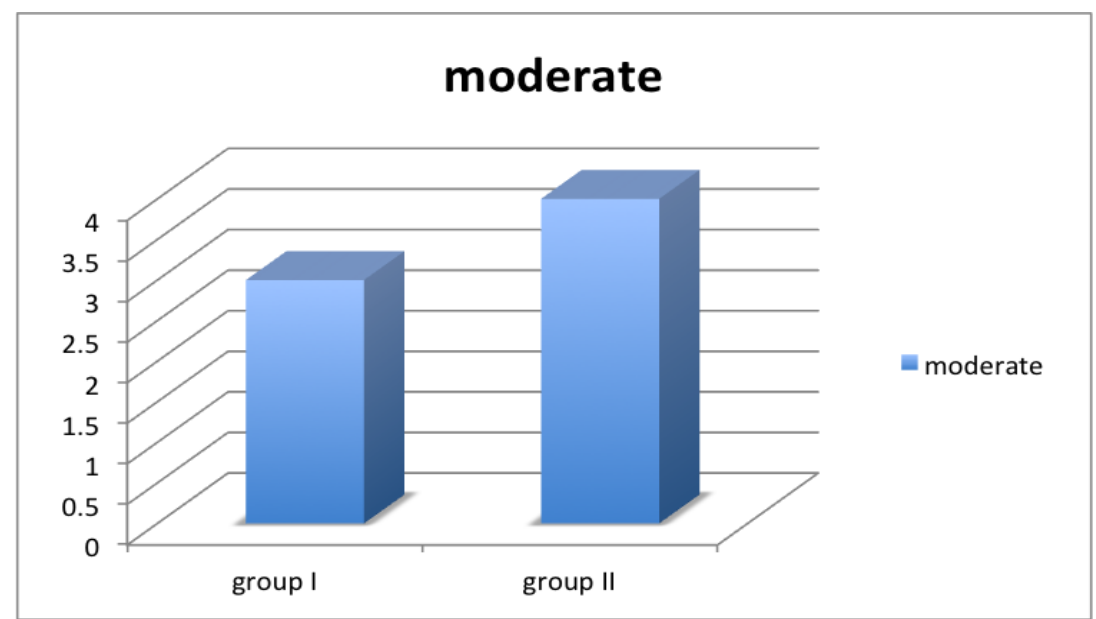

(Fig.5) Comparing moderate TMD among males and females

\section{Conclusion}

When comparing group I and group II, untreated patients with class I and class II in group I have only $2 \%$ of moderate TMD whereas in group II patients who had underwent orthodontic treatment to treat class I and class II malocclusion had $3 \%$ moderate TMD. It has been concluded from this study that patients who had underwent orthodontic treatment are more prone to TMD when compared to untreated patients with class I and class II malocclusion due to improper occlusal contact after orthodontic treatment. This study is insufficient to prove that patients who had underwent orthodontic treatment are more prone to TMD because of small sample size.

\section{Reference}

[1]. Relationship Between Signs and Symptoms of Temporomandibular Disorders and Orthodontic Treatment: A Cross-sectional Study. Ana Conti, DDS, MS ${ }^{\mathrm{a}}$; Marcos Freitas, DDS, PhD ${ }^{\mathrm{b}}$; Paulo Conti, DDS, $\mathrm{PhD}^{\mathrm{c}}$; José Henriques, DDS, $\mathrm{PhD}^{\mathrm{d}}$; Guilherme Janson, $\mathrm{DDS}, \mathrm{PhD}$

[2]. 1. Okeson JP. Orofacial Pain. Guidelines for Assessment, Diagnosis and Management. Chicago, Ill: Quintessence Pub; 1996:113184. 2. Costen JB. A syndrome of ear sinus symptoms dependent upon

[3]. Disturbed functions of TMJ. Ann Otol (St Louis). 1934;43:1-15. 3. Costen JB. Neuralgias and ear symptoms associated with disturbed function of the TMJ. J Am Med Assoc. 1936;107:252-254

[4]. Wyatt WE. Preventing adverse effects on the temporomandibular joint through orthodontic treatment. Am J OrthodDentofacial Orthop. 1987:91:493-499.

[5]. Nielsen L, Melsen B, Terp S. TMJ function and the effects on the masticatory system on 14-16-year-old Danish children in relation to orthodontic treatment. Eur Orthod. 1990;12:254-262.

[6]. Conti PCR, Ferreira PM, Pegoraro LF, Conti JV, Salvador MCG. A cross-sectional study of prevalence and etiology of signs and symptoms of temporomandibular disorders in high school and university students. J Orofac Pain. 1996;10:254-262.

[7]. Fonse^ca DM. Disfunc a o Craniomandibular (DCM) — diagnósticopelaanamnese[master's thesis]. Bauru: Bauru School of Den tistry, University of Sã o Paulo; 1992.

[8]. Helkimo M. Studies on function and dysfunction of the masti- catory system. III-analysis anamnetic and clinical recordings of dysfunction with the aid of indices. Swed Dent J. 1974;67:15-82.

[9]. Bales JM, Epstein JB. The role of malocclusion and orthodontics in temporomandibular disorders. J Can Dent Assoc. 1994;60: 899 -905 .

[10]. Temporo mandibular disorder and orthodontic treatment - A review with a reported clinical case Tomislav Badel, MiljenkoMarotti and Ivana Savic Pavicin. 